\title{
Por los entrecruces del género, la justicia y la legalidad en tierras indígenas
}

\author{
Rosalva Aída Hernández Castillo
}

MARÍA TERESA SIERRA (coord.), 2004

\section{Haciendo justicia. Interlegalidad, derecho y género en regiones indígenas}

Centro de Investigaciones y Estudios Superiores en Antropología Social, Miguel Ángel Porrúa, Cámara de Diputados-LIX Legislatura, México.

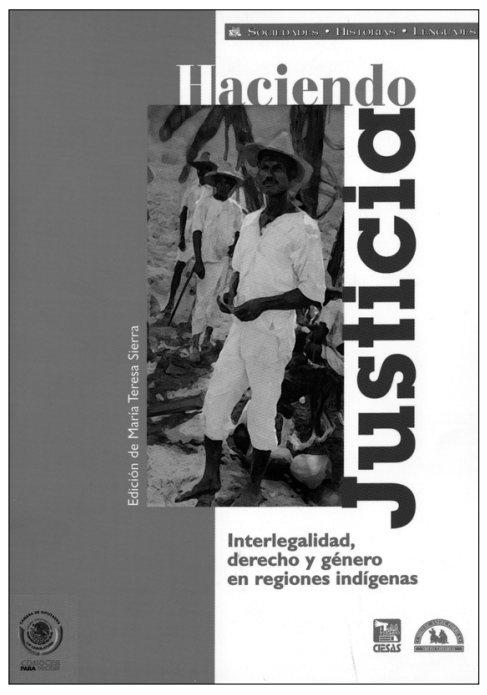

$H$ aciendo justicia. Interlegalidad, derecho y género en regiones indígenas es el producto de un trabajo de investigación colectiva de tres años que se propuso comparar los espacios de interlegalidad y los usos del derecho por parte de hombres y mujeres indígenas en seis regiones de México. Pero más allá de esto, es resultado de un largo proceso de investigación y reflexión teórica que Teresa Sierra y Victoria Chenaut vienen realizando desde la década de 1980, confrontando visiones dicotómicas e idealizadas del derecho indígena. Pioneras en la

Rosalva AÍdA HeRnández CASTILLO: Centro de Investigaciones y Estudios Superiores en Antropología SocialDistrito Federal, México.

aidaher2005@yahoo.com.mx

Desacatos, núm. 23, enero-abril 2007, pp. 325-329. antropología jurídica mexicana, estas dos autoras han contribuido a la formación de una nueva generación de antropólogas preocupadas por el entrecruce entre la legalidad, la cultura y el poder, cuya rigurosidad etnográfica y calidad académica se pone de manifiesto en este libro.

Esta antología da continuidad al ya clásico volumen editado por ambas en 1995, Pueblos indígenas ante el derech $o^{1}$, referencia obligada para quienes trabajamos el entrecruce entre el derecho y el poder en el México indígena. La preocupación por la interlegalidad planteada en aquel volumen se ve desarrollada en este nuevo proyecto retomando como eje analítico la propuesta teórica de Boaventura de Souza Santos de considerar que "las

\footnotetext{
${ }^{1}$ CIESAS y CEMCA, México.
} 


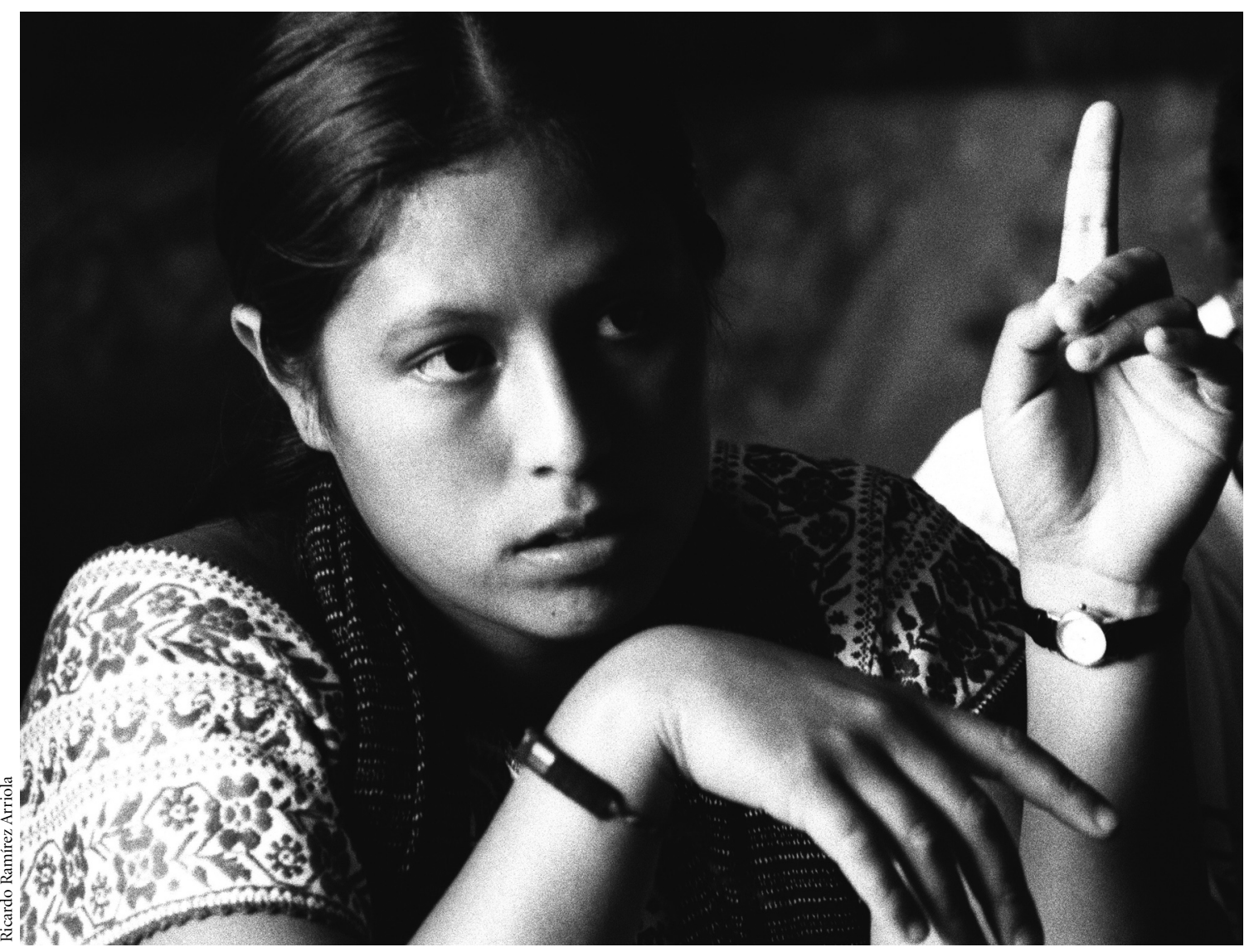

normas del derecho estatal y las normas del derecho indígena se encuentran imbricadas, constituyéndose mutuamente en formas que implican conflicto normativo, pero también negociación y múltiples transacciones" 2 .

Ya desde la década de 1980 Teresa Sierra confrontó las visiones dicotómicas del derecho y la costumbre, presentes en el discurso académico y político de la época, que ponían énfasis en la exclusión que el Estado mexicano había hecho de los pueblos indígenas y reivindicaba la existencia de un derecho indígena antagónico al derecho nacional. Las concepciones funcionalistas de la ley y la costumbre que prevalecieron en los estudios de antropología jurídica hasta la década de 1980 seguían con-

\footnotetext{
2 Victoria Chenaut, "Prácticas jurídicas e interlegalidad entre los totonacas del Distrito Judicial de Papantla”, en el libro aquí reseñado, p. 291.
}

cibiendo el espacio legal como una esfera independiente, factible de analizarse al margen de otros procesos económicos y sociales. Tanto los promotores del análisis de sistemas normativos, herederos de la tradición metodológica de Radcliffe-Brown, como quienes reivindicaban el análisis de los procesos jurídicos, siguiendo a Malinowski, ignoraban la manera en que los sistemas o procesos analizados se imbricaban y constituían mutuamente.

El desarrollo de la propuesta articulacionista hecha por Teresa Sierra vino a cuestionar tanto las perspectivas funcionalistas que ignoraban la manera en que los procesos jurídicos analizados se insertaban en relaciones coloniales o poscoloniales de dominación, como las perspectivas de la antropología crítica mexicana, que veía a ambos derechos como antagónicos y excluyentes. $\mathrm{Su}$ propuesta metodológica enfatizaba la articulación entre sistemas normativos dominantes y dominados, a partir 
de estrategias desarrolladas por los indígenas al recurrir a una $\mathrm{u}$ otra instancia ${ }^{3}$. Ahora Victoria y Teresa, uniendo sus reflexiones a las de Jane Collier, Ivette Vallejo, Elisa Cruz, Maribel Nicasio y Rebeca Igreja, van más allá de este análisis articulacionista y nos muestran cómo el llamado derecho indígena y el derecho nacional no coexisten con articulaciones muy precisas, sino que los contactos afectan el contenido mismo de las normatividades, proponiéndonos la existencia de una mutua constitución de ambas legalidades.

Esta propuesta resulta especialmente audaz en un momento político en el que la reivindicación de un derecho propio ha sido central en las demandas autonómicas de los pueblos indígenas. Sin embargo, su llamado a considerar los sistemas normativos indígenas como entidades dinámicas y flexibles, insertas en procesos históricos de poder y de cambio sociojurídico, hace eco a las perspectivas anti-esencialistas que desde el movimiento indígena han hablado de la necesidad de "cambiar permaneciendo y permanecer cambiando", me refiero a las voces de las mujeres indígenas organizadas que han hecho un llamado a reinventar las tradiciones desde perspectivas más incluyentes y justas, para todos y todas las integrantes de los pueblos indígenas.

Esto nos lleva al segundo aporte fundamental de este libro, la perspectiva de género, que nos muestra de manera comparativa cómo hombres y mujeres indígenas hacen un uso diferenciado de los espacios propios de impartición de justicia y del derecho nacional. Mediante una detallada etnografía de los procesos de disputa entre nahuas de la Sierra Norte de Puebla; totonacas de la Sierra de Papantla, Veracruz; mixes de Totontepec, Oaxaca; tzotziles de Zinacantán, Chiapas; mixtecos de la montaña de Guerrero y mazahuas, otomíes y triquis de la ciudad de México, las autoras de esta colección nos muestran cómo las desigualdades de género han marcado los problemas de acceso a la justicia que tienen las mujeres indígenas tanto frente a las autoridades tradicionales como ante el ministerio público. Las voces de las

${ }^{3}$ Véanse María Teresa Sierra, "Usos y desusos del derecho consuetudinario”, Nueva Antropología, vol. XIII, núm. 44, 1993, pp. 20-39; y el libro de Sierra y Chenaut antes citado. mujeres nahuas de Cuetzalan, Puebla, registradas en el excelente artículo de Ivette Vallejo, o de las mujeres tzotziles de Zinacantán, revisitadas por Jane Collier, nos recuerdan que el llamado derecho indígena no es la panacea para los problemas de violencia y exclusión que viven las mujeres indígenas, pero tampoco lo es el derecho nacional, con sus ministerios públicos racistas y misóginos, como lo prueban las experiencias de las mujeres indígenas migrantes en la ciudad de México entrevistadas por Rebeca Igreja o las mujeres totonacas de Coyutla, que con gritos e insultos rechazan las decisiones legales que les son adversas, de las cuales nos habla Victoria Chenaut.

En especial el trabajo de Rebeca Igreja pone en evidencia el racismo que permea a los órganos de impartición de justicia, un racismo estructural que se manifiesta no sólo en el trato que reciben los indígenas por parte del Ministerio Público, sino en la legalidad misma, que parte de una visión liberal e individualista de los derechos que no deja espacio para repensar a la ciudadanía desde la especificidad cultural. A pesar de los cambios promovidos por los gobiernos perredistas, reconocidos por Igreja y que incluyen programas especiales de defensoría legal, apoyo a proyectos productivos, programas de asesoría a padres para evitar la violencia infantil y la drogadicción juvenil, la violencia estructural de la marginación urbana, el desempleo y la mendicidad, la prostitución infantil y la drogadicción son el marco en el cual indígenas otomíes, triquis y mazahuas intentan reconstruir los espacios propios de impartición de justicia, muchas veces con poca legitimidad frente a las nuevas generaciones, que han aprendido a vivir temiendo a la justicia del Estado e ignorando la justicia comunitaria.

Desde los barrios marginales de la ciudad de México hasta las comunidades aisladas de la montaña de Guerrero, las historias de vida, los archivos judiciales, las etnografías de los procesos de disputa que documentan los distintos artículos nos hacen ver cómo los sistemas hegemónicos sexo-género marcan la manera en que se concibe la justicia en las distintas regiones indígenas. En los largos procesos conciliatorios que nos documentan Jane Collier, Teresa Sierra, Ivette Vallejo y Victoria Chenaut, las autoridades tradicionales les recuerdan a los hombres y mujeres indígenas sus roles de género: ellos deben ser 
buenos proveedores y ellas deben atender y cuidar a la familia, encargándose del espacio doméstico. A las mujeres se les reconcilia con sus esposos, pero también con el lugar de subordinación que muchas de ellas tienen en sus comunidades. Esta comprobación no lleva a las autoras a concluir de manera simplista que, entonces, tanto el derecho nacional como el indígena son lo mismo: herramientas de dominación que reproducen estructuras patriarcales. Varias de las autoras, y en especial Jane Collier, reivindican los procesos conciliatorios de las comunidades indígenas con todas sus desigualdades y contradicciones, como más respetuosos del sentir de las mujeres indígenas, en comparación con los procesos nacionales, en los que los ministerios públicos no hablan su lengua, no las escuchan y muchas veces las "regañan" con actitudes irrespetuosas y racistas. Los trabajos mantienen siempre la tensión entre una perspectiva del derecho como reproductor de las relaciones de poder y como herramienta de resistencia. A pesar de todas las limitaciones que enfrentan, las mujeres indígenas son importantes usuarias de los espacios nacionales de justicia. La alternancia del derecho les abre otras posibilidades de justicia: cuando la justicia comunitaria les es adversa, muchas mujeres indígenas recurren al derecho nacional para renegociar sus relaciones, rechazar imposiciones o ganar derechos. La apuesta de las autoras, por lo menos de algunas, consiste en reconocer la existencia de lógicas culturales distintas que permiten resolver problemas con base en conciliaciones, pero dejar siempre la puerta abierta a otros espacios de justicia. Esta apuesta conlleva algunas contradicciones, que se abordan en el artículo de Teresa Sierra, y es que en la medida en que más demandantes indígenas utilizan el derecho nacional para renegociar acuerdos comunitarios se debilitan los espacios de jurisdicción indígena. ¿Cómo apoyar el reconocimiento de un derecho propio sin descartar las posibilidades de tener acceso a la justicia del Estado? Éste es el dilema político que enfrentan quienes reivindican estas perspectivas más complejas del derecho indígena.

A diferencia de aquellos que han señalado las contradicciones internas que marcan la vida cotidiana de las comunidades indígenas como un argumento para negar la viabilidad de los proyectos autonómicos, detrás de los análisis que presenta esta antología parece subyacer la convicción de que el reconocimiento de la manera en que las relaciones de poder marcan las subjetividades no niega la posibilidad de construir, con base en esas conciencias contradictorias, nuevos imaginarios colectivos. Las mujeres indígenas, en diversas regiones del país, están dando una lucha por repensar el derecho indígena desde perspectivas más incluyentes, como nos lo demuestran Ivette Vallejo y Teresa Sierra, y como lo hemos señalado en otros trabajos quienes analizamos los procesos organizativos y políticos de las mujeres indígenas. Queda aún por evaluar qué implicaciones tiene para las "lógicas culturales" el repensar las subjetividades indígenas y las relaciones entre los géneros bajo nuevos términos.

Es en este punto que surgen algunas de mis dudas con respecto a los análisis presentes en este libro, sobre todo en lo que respecta al trabajo de Jane Collier. De todas las autoras de esta colección ella es quien más se preocupa por demostrar que existen lógicas culturales distintas entre los zinacantecos y los encargados de los órganos de justicia que no son indígenas. Varias de las autoras hacen eco a esta afirmación en sus análisis de los procesos de conciliación promovidos por las autoridades indígenas; sin embargo, también nos muestran cómo los funcionarios locales encargados de los procesos de impartición de justicia comparten muchas veces perspectivas conciliatorias y del "deber ser" femenino y masculino de los usuarios indígenas. Ésta ha sido mi experiencia en la zona mam de la Sierra Madre de Chiapas, donde la población mestiza comparte muchas de las lógicas culturales de los campesinos indígenas, los ministerios públicos no indígenas temen ser embrujados por los demandantes inconformes y comparten la perspectiva de que los jóvenes que se "fugan" deben de pagar una "multa" a sus padres para ser perdonados. En muchos sentidos sus "lógicas culturales” son más cercanas a las de la población mam que a mi "lógica cultural de mestiza". Los funcionarios encargados de la justicia del Estado son también producto de procesos culturales complejos que no se reducen a su formación como especialistas del derecho nacional. Con esto quiero apuntar es que la existencia de lógicas culturales distintas entre indígenas y mestizos tiene que ser un punto de llegada y no de partida, para no 
reproducir nuevamente visiones dicotómicas y orientalizantes de las culturas indígenas.

Otra de las dudas que me surgen del trabajo de Jane Collier tiene que ver con un énfasis excesivo que ella pone en diferenciar entre un pasado más armónico en las relaciones de género entre los zinacantecos que contrasta con un presente de exclusión y pérdida de poder por parte de las mujeres. Su trabajo analiza los cambios que ha sufrido el derecho zinacanteco en las últimas tres décadas, a partir de que la agricultura de autosubsistencia se ve desplazada por los nuevos mercados laborales. $\mathrm{Su}$ argumento principal es que la introducción de relaciones capitalistas en esa región ha transformado las relaciones de género, sustituyendo los roles de complementariedad, característicos de las economías de autosubsistencia, por relaciones más desiguales. Desde estas perspectivas, en el nuevo contexto económico las mujeres pierden autonomía al depender más de los salarios de sus esposos o sufrir una inserción marginal en el mercado capitalista. Es difícil contra-argumentar análisis de quien ha sido testigo y acompañante por casi cuarenta años de los cambios vividos en esa comunidad indígena. Sus propios trabajos y los de otros antropólogos del proyecto Harvard que trabajaron en los Altos de Chiapas en la década de 1960 son nuestra referencia para reconstruir lo que pasaba entre los hombres y mujeres tzotziles en esos años, y es importante recordar que el paradigma funcionalista que hegemonizaba el análisis antropológico tendía a enfatizar las relaciones armoniosas silenciando el conflicto y las relaciones de poder. ¿Hasta qué punto no fueron las lentes con las que Jane Collier veía la realidad en la década de 1960 las que la hicieron ignorar la violencia doméstica o los conflictos maritales? ¿Las relaciones de complementariedad de las economías de autosubsistencia no fueron más un mito construido por los antropólogos funcionalistas que una realidad vivida por las mujeres? Los testimonios desgarradores de las ancianas tzotziles, que fueron las cabezas de familia de la década de 1960 y que han reproducido etnografías recientes como las de Graciela Freyermuth y Anna María Garza, parecen apuntar en ese sentido.

Más allá de la discusión sobre si las mujeres indígenas están peor ahora que antes de que se insertaran de ma-

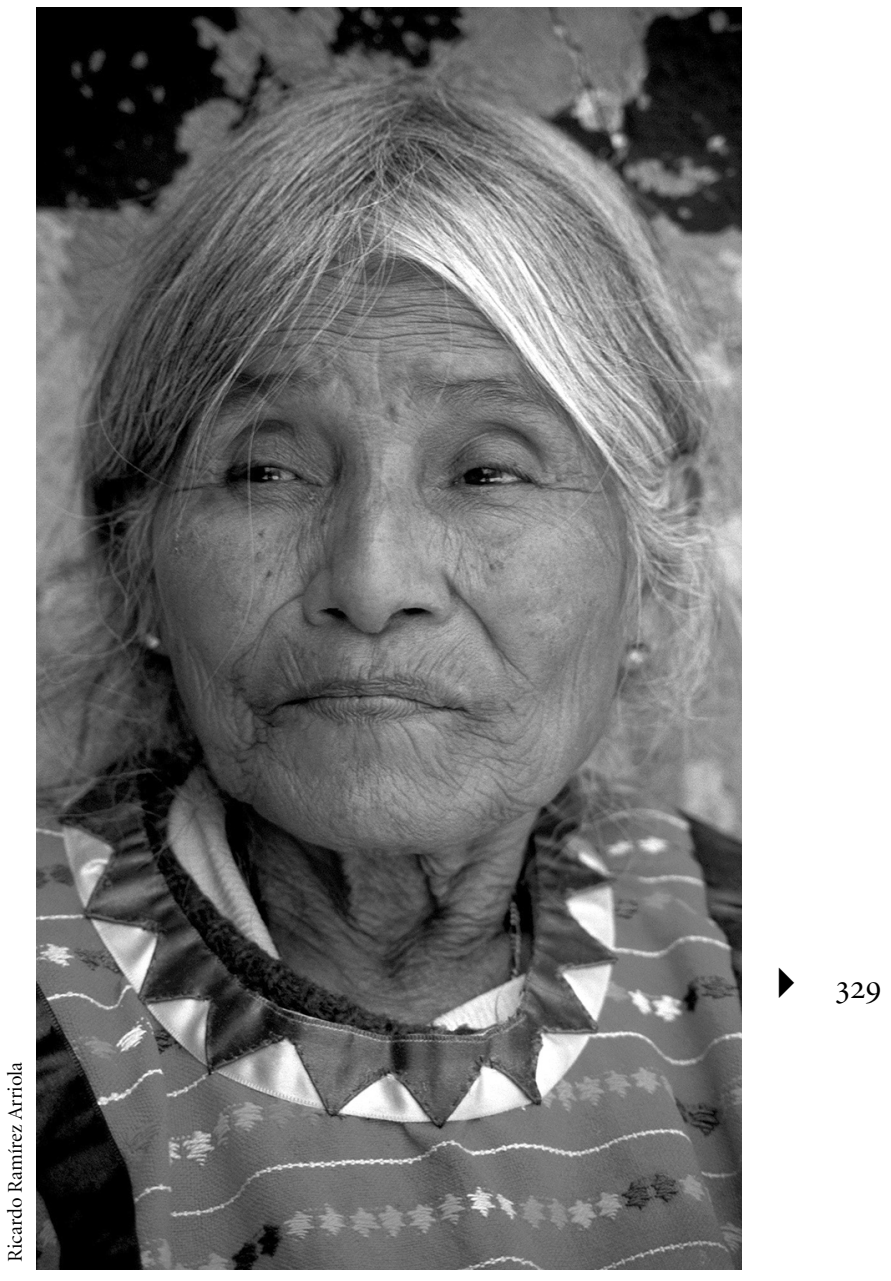

nera más directa en el mercado laboral, lo que este libro abre es un nuevo debate en torno a cómo repensar el derecho desde el género y cómo repensar el género desde la cultura. En este sentido, es ya un texto imprescindible para quienes enseñamos o investigamos sobre temas relacionados con los estudios de género, la antropología jurídica y la cuestión étnico nacional. Les agradecemos a Teresa Sierra, Jane Collier, Ivette Vallejo, Victoria Chenaut, Elisa Cruz, Maribel Nicasio y Rebeca Igreja la profundidad de sus análisis y la densidad de sus etnografías. Sus trabajos me han ayudado a entender un poco más los retos y posibilidades que ofrece el derecho.

Septiembre de 2005 